\title{
ASPECTOS EPIDEMIOLOGICOS DE Botrytis cinerea Pers. EN CHIRIMOYOS (Annona cherimolia Mill.)
}

\author{
(Epidemiological aspects of Botrytis cinerea in custard apple-tree \\ (Annona cherimolia Mill.))
}

\author{
Verónica Cotroneo Z. \& Ximena Besoaín C. \\ Facultad de Agronomía, Universidad Católica de Valparaíso. \\ Casilla 4-D, Quillota, Chile.
}

Palabras clave: Botrytis cinerea, cpidemiología, chirimoyos.

Key words: Botrytis cinerea, epidemiology, custard apple-trce.

\section{RESUMEN}

Debiclo a que Botrytis cincrea causa importantes pérdiclas en pre y postcosecha en chirimovas, se realizó un seguimiento de un huerto én producción en la localidad de La Palma, Quillota, para determinar su incidencia, correlacionándola con la temperatura, humedad relativay precipitación. Para determinar la presencia de este hongo, se realizó un muestreo de hojas, fiutos y flores tardias entre abril y octubre de 1997. Estas muestras se colocaron en cámaras himedas por 10 dias, bajo condiciones de luz y temperatura favorables a la esporulación.

En octubre y noviembre de 1997, se determinó su presencia en semillas y en 66 firutos (embaladosy mantenidos a temperatura ambiente por 12 dias) y en febrero de 1988 su presencia en flores. Todas las cepas obrenidas se sembraron en APD a $22^{\circ} \mathrm{C}$ por 10 dias.

La incidencia de cepas de Botrytis comenzó en el periodo de precipitaciones y fire en aumento después de los meses más lluriosos. La primera determinación en hojas, con o sin sintomas, se inició en mayo y logró un máximo en octubre. En junio comenzé su detección en frutos logrando su máximo én septicmbre, no evidenciándose sintomas de pudrición én éstos. No se detectó Botrytis en flores, flores tardias ni en semillas. La incidencia de Botrytis en frutos en postcosecha alcanzó un 10,6\%.

De 9 aislamientos de Botrytis efectuados se determinó sólo la presencia de B. cincréa.

\section{INTRODUCCIÓN}

Botrytis cincrea, agente causal de pudrición gris en chirimoyas (Annona cherimolia Mill.), es uno de los géneros que causa mayores pérdidas en almacenạje y en la

\section{SUMMARY}

Since Botrytis cinerea causes important loses in pre and post-harvest period of custard apples-tree, a prospect was done in a custard apple-tree orchard in the locality of La Palma, Quillota, to determine the presence of this fungus: Samples of leaves, fiuits and off season flowers were taken between april and october of 1997. These samples were deposited in a humid chamber, under light and temperature cunditions favorable for sporulation. In october and november of 1997, the presence on seeds and 66 fruits (packed and maintained at room temperature for 12 days) and its presence in flowers on february 1998 was also determined.

The incidence of Botrytis began when the rainy period starts and increases its presence after this period finishes: The first determinations of $B$. cincrea in leaves with or without symptoms began in mav, with a maximum of detection in october. In june began the detections on fruits with a maximum in september: No evidence of decay was detected in the fruit collected in this period. Botrytis was not detected in flowers, off season flowers or seeds. The incidence of Botrytis in post-harvest fruits achieved a 10,6\%. Of nine isolates analyzed only the presence of B. cinerea was determined.

etapa de comercialización. Esta fruta es altamente perecible y no se ha desarrollado hasta el momento un sistema de conservación por largos períodos, por lo que se exporta vía aéréa, salvo a Argentina (INTEC-CHILE, 1985).

Muchos estudios se han realizado en epidemiología de Botrytis, pero en la familia Annonaceae 
nada se ha investigado. Debido a la importancia de la pudrición gris en la perecibilidad de las chirimoyas, tanto en postcosecha como en precosecha, resulta necesario conocer su comportamiento en los distintos estadíos fenológicos respecto a las condiciones ambientales, a modo de estudio preliminar, para investigaciones más específicas en csla matcria.

De acuerdo con trabajos realizados en otros frutos, se cstableció que la tempcratura juega un rol importante para el desarrollo de la pudrición gris. Las bajas temperaturas provocan estrés por congclamiento, aumentando la susceptibilidad de la planta; $y$ las altas tempcraturas llevan a una ruptura de la resistencia normal de los tejidos del hospedero (Jarvis, 1992).

Otro factor implicado en el desarrollo de Botrytis es la humedad. La infección pucde ocurrir a bajjas temperaturas si las plantas permanecen mojadas largo tiempo (Rics. 1995).

En cuanto a las fucntes de inóculo, Braun \& Sulton (1987), obscrvaron que cl $99 \%$ del inóculo de $B$. cinerea en frutillas, fue producido a partir de micelio en la lámina y pecíolos de hojas mucrlas y muy poco de coronas y frutos momificados.

De acuerdo con los antecedentes antes expuestos, este cstudio tuvo como objetivo determinar la incidencia de Botrytis en los distintos órganos del chirimoyo y correlacionarla con temperaltura. humedad relativa, y precipitación.

\section{MATERIALES Y METODOS}

El trabajo se realizó en un hucrto de chirimoyo perteneciente a la Estación Experimental La Palma, Facultad de Agronomía, Universidad Católica de Valparaíso, ubicado en la localidad de La Palma, Quillota, V Región. Las variedades mucstreadas corresponden a Bronccalda y Concha lisa. Las muestras se analizaron en cl Laboratorio de Fitopatología de la Facultad de Agronomía de la Universidad Católica de Valparaíso.

\section{Ensayyo de evaluación epidemiológica.}

Los muestreos fueron efectuados entre los meses de abril a octubre de 1997, debido a que cn octubre los árbolcs renucvan su follaje. La melodología emplcada fue la siguiente: se seleccionaron alcatoriamente dos hilcras del huerto, muestreando un total de 25 árboles al azar, a los que se les sacó dos hojas en lo posible con síntomas, de lo contrario se tomó cualquier hoja al azar. De cinco diferentes árboles se cetrajo un fruto, $y$ si habia flores se tomaron 20 por muestreo. Esto se hizo dos veces por semana, recogiendose un tolal semanal de $1(0)$ hojas. 10 frutos y to flores.
Las muestras colectadas entre abril y octubre se colocaron por espacio mínimo de 10 días en cámaras húmedas, mediante el empleo de contenedores de plástico transparente de $15 \times 10 \mathrm{~cm}$, desinfectadas con alcohol, rotuladas con la fecha de recolección y un número correlativo para cada muestra. Cada una contenía toalla Nova estéril humedecida con agua destilada estéril (ADE) donde se colocaron ya sea una a dos hojas, uno a dos frutos, o scis flores, procurando que estas estructuras no se toparan excesivamente. Para favorecer la esporulación, se mantuvicron en una sala calcfaccionada $\left(2()-22^{\circ} \mathrm{C}\right)$ y con luz artificial por las noches. Finalmente se revisó cada cámara con la ayuda de una lupa estercoscópica y microscopio óptico si cra necesario.

En algunos casos las mucstras que presentaban micelio y conidios del género Botrytis, fucron repicadas y purificadas en medio agar papa dextrosa acidulado (APDA), a un pH 5,5. El hongo aislado fue guardado en tubos de ensayo con $\mathrm{ADE}$ y refrigerados a $5^{\circ} \mathrm{C}$ para su posterior identificación.

La decrminación de la incidencia de Botrytis se realizó mediante la observación de micclio y esporas en cámaras húmedas, y aislamiento en medio de cultivo APDA. Se calculó la incidencia de Botrytis en hojas y frutos de de chirimoyo de acuerdo a las siguientes fórmulas:

\section{Incidencia de Botrytis en hojas}

$=\mathrm{N}^{\circ}$ de árboles con presencia de Botrvtis en hojas $\mathrm{N}^{\circ}$ total de árboles muestreados

\section{Incidencia de Botrytis en frutos}

$$
=\frac{\mathrm{N}^{\circ} \text { de frutos con presencia de Botrytis }}{\mathrm{N}^{\circ} \text { total de frutos muestreados }}
$$

Evaluación de incidencia de Botrytis en semillas.

El ensayo se realizó en octubre para determinar en forma aproximada la presencia de Botrytis en las semillas. Se colcctaron, al azar, scis frutos por semana, durante tres semanas consecutivas. Despućs de recolecladas las semillas, se lavaron en hipoclorito de sodio al $1 \%$ por 15 segundos, se enjuagaron y secaron bajo campana de flujo laminar, se cortaron en mitades y se sembraron en medio APDA, incubandose por 10 días a $22^{\circ} \mathrm{C}$.

\section{Evaluación del daño de firutos en postcosecha.}

Se hizo un seguimicnto a 66 frutos maduros, aparentemente sanos. Éstos fueron cosechados el día 19 y embalados cl 22 de noviembre de 1997, posteriormente fueron craluados el 2 de diciembre. Para simular las condiciones a las que se somete la fruta en la posicosecha se realizó cl procedimiento de embalaje y almaccnamiento de acuer- 
do con las exigencias para la cxportación. Los frutos se limpiaron con una brocha, sumergićnclose por 22 segundos en Deterfrut neutro. diluido en agua $(1: 30(0))$ y secados con toalla nova cstćril. Sc puso cada fruto en un soquetc slivit y se colocaron cn cajjas de cartón de $4-4,5 \mathrm{~kg}$. perforadas para su ventilación. Éstas se mantuvieron a temperatura ambiente por 12 días y se craluaron midiendo $\mathrm{cl}$ porcentaje de fruta con pudrición gris.

\section{Evaluación de incidencia de Botrytis en flores.}

El cstudio se realizó durante febrero de 1998, para determinar. durante cl periodo de plena floración, la presencia o ausencia de Botrytis cn flores. Se colectaron, al azar 50 flores por mucstreo. dos veces por semana, analizándose un total de 4()() flores. Éstas se cortaron por la mitad, se lavaron en hipoclorito de sodio al $1 \%$ por espacio de 15 segundos y luego se enjuagaron en agua destilada estéril. Se secaron y sembraron cn APDA y se mantuvieron cn estufa a $22^{\circ} \mathrm{C}$ por 10 días. craluándose la presencia de Botrytis luego de esle período.

\section{Identificación de eepas de Botrytis obtenidas.}

Para identificar las cepas de Botrytis se usó las claves descritas por Ellis (1971) y Domsch et al.,(1980). Se midió al microscopio óptico el largo y ancho de los conidios y conidióforos de 9 aislamicntos (de un total de 15), obtenidos cntre abril y octubre de 1997. Las mediciones fueron efectuadas a partir de cultivos en agar arcena(Oatmeal agar) a $22^{\circ} \mathrm{C}$. incubados durante 2 semanas. En cadla una de ćstas se midió un total de 50 conidios y 10 conidióforos.

\section{RESULTADOS Y DISCUSION}

a) Incidencia de Botrytis cinerea en huerto de chirimoyo. De acuerdo con los resultados obtenidos a partir de las muestras de hojas, frutos y flores tardias colocadas en cámlaras húmedas. se aprecia que en chirimovos, Botrytis fue detectado inicialmente en hojas y un mes más tarde en frutos (Figura I). En ninguno de los muestreos de flores fucra de ćpoca, realizados durante cl otoño de 1997 se observó presencia de esic hongo.pero fue decectado tanto en hojas asintomáticas y sintomálicas (Figura 2c), micntras que todos los frutos colectados se cncontraban sin síntomas. Sólo al final del cultivo fue posible apreciar frutos con moho grisícco (Figura 2d). Las hojas con síntomas se caracterizaron por la presencia de un atizonamiento de color caré claro, el que se formaba a partir del margen de ćstiss, invaginándose hacia el centro de la lámina, detectándose. bajo condiciones de alta humediad, halos concéntricos y la prescncia del moho pardo - grisácco (Figura 2c).

En ninguna de las muestras colectadis en el mes de abril se desarrolló Botrytis cn cámara húmeda. La incidencia de Botrytis en hojas comenzó 10 dias antes de la primcra lluvia en mayo, con una baja incidencia (3\%) (Figura 1). Al observar los datos de humedad y temperatura mínima, se deduce que debió haber condensación sobre los tejidos foliares, pues la humedad relativa se mantuvo muy ccrcana al $100 \%$ y las temperaturas minimas fluctuaron entre los $5^{\circ}$ y $11^{\circ} \mathrm{C}$, con una media de $7^{\circ} \mathrm{C}$. Esta condensación probablemente formó una película de agua necesaria para la germinación del hongo ( Kosuge \& Hewitt, 1964). En cl último tercio del mes aparecen las lluvias y la incidencia baja a ccro hasta cl final de la primera semana de junio. Esta baja podría deberse a que despućs de lluvias intensas y duraderas, disminuye la cantidad de conidios atmosfóricos (Cortesi, et al., 1991). Efectivamente, hubo lluvias intensas, con precipitaciones de $20,5 \mathrm{~mm}$ el día 18 de mayo, $+1 \mathrm{~mm} \mathrm{cl} 29$, y $60 \mathrm{~mm}$ al día siguicnte. Esto probablementc favorece una baja cantidad de inóculo aéreo, por tratarse de un ciclo infeccioso incipiente.

Durante junio (Figura 1), la incidencia de Botrytis se mantiene alrededor del $3 \%$, constante en todos los muestreos. Las condiciones ambientales fucron: humedad relativa de $80 \%$ a $100 \%$, temperaturas máximas cercanas a $17^{\circ} \mathrm{C}$, tcmperaturas mínimas medias de $6^{\circ} \mathrm{C}$ y precipitaciones frecuentes pero menos intensas que cl mes anterior (la máxima precipitación en un día fuc de $26,5 \mathrm{~mm}$ ). Las precipitaciones son bastante favorables a la infección, a pesar de las bajaias temperaturas medias diarias. Scgún Ries (1995), la infección puede ocurrir a bajas temperaturas cuando las plantas permanecen mojadas por largos períodos de ticmpo. Nelson(1951), scñala que cn uva de mesa son necesarias 72-84 horas de agua libre, a $3^{\circ} \mathrm{C}$ para que se produzca la infección, además se favorece la dispersión de $\mathbf{B}$. cinerea. Cortesi et al. (1991), atribuyen a la lluvia el principal factor de dispersión de conidios dependiendo de su frecuencia e intensidad. Durante este mes se observa por primera vez presencia de Botrytis en tejidos de frutos con una incidencial de unl 5\% (Figura 1).

Entre fines de junio e inicios de julio (Figura l), hubo heladis por tres días consecutivos, lo que aumenta la susceptibilidad al ataque del palógeno, por la ruptura de cćlulas y daño de los tejidos (Jarvis, 1992). Esto, sumado a que Botrytis es un palógeno que causa enfermedades. multicíclicis. cxplicaría la incidencia del $21 \% \mathrm{en}$ el follaje durante julio y la constante presencia del hongo en todos los mucstreos analizados. Las temperaturas después de las heladas se mantuvieron similares a las del mes anterior. La humedad relativa aumentó a un rango de $90 \%$ a $100 \%$ y solo hubo precipitaciones durante tres días en $\mathrm{cl}$ mes. Probablemente la cantidad de inóculo aćreo aumentó por las reinfecciones. La incidencia promedio de Botrytis $\mathrm{cn}$ tçidos de frutos fue de un $8 \%$ durante julio. 


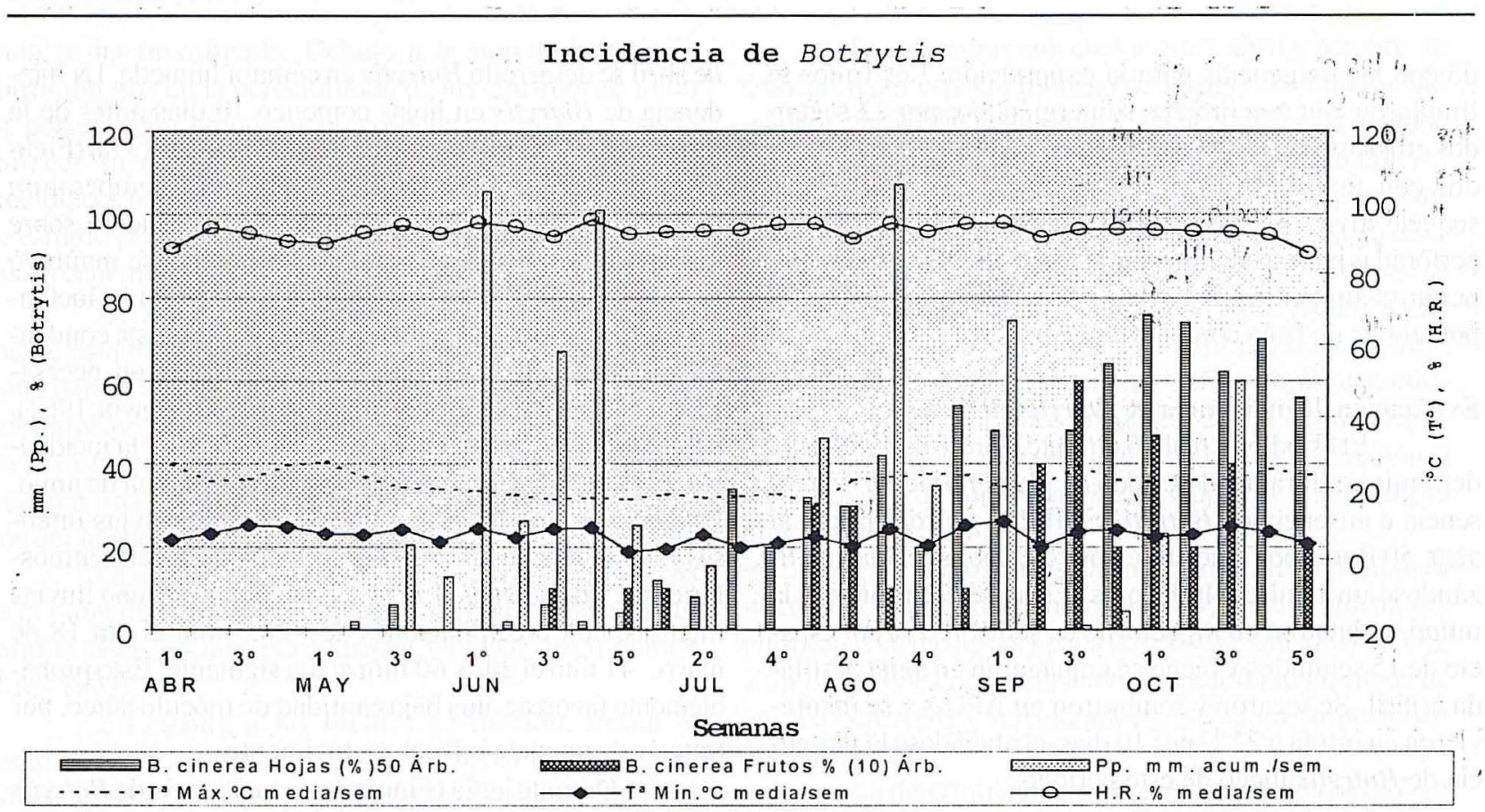

Figura 1.- Presencia de B. cincrea en chirimoyos en tejidos de hojas y frutos.

(1): Quillota, Abril a Octubre de 1997.

En agosto. las temperaturas máximas medias ascendieron a $22^{\circ} \mathrm{C}$, las temperaturas medias aumentaron a $14^{\circ} \mathrm{C}$ y se obscrvó dos heladas durante la primera scmana. La humedad relativa se manturo similar a la del mes anterior y las precipitaciones se concentraron en la mitad del mes. Se produjo una cxplosión en la incidencia de Botrytis, probablemente por el aumento del inóculo debido a las rein-fecciones y a los daños provocados por las heladas, adcmás de las temperaturas máximas promedio de este mes. que favorecen el crecimiento del micelio. Al parecer, la temperatura óptima para el crecimicnto micclial es de $22^{\circ} \mathrm{C}$. (Jarvis, (1977a, cilado por Jarvis , 1992), micntras que para la esporulación. fluctuaría entre $15^{\circ}$ y $22^{\circ} \mathrm{C}$ (Sosi-Alvarez. et al.,1995) y para la germinación scría de $20^{\circ} \mathrm{C}$ (Jarvis ,1992). La incidencia de Botrytis en chirimoyos. craluada en los tcjidos de las hojas. aumenta a un 36\%, y en los frutos a un 12\% (Figura 1).

En septicmbre se mantienen las condiciones ambientales del mes anterior. la temperatura media llegó a $15^{\circ} \mathrm{C}$ y aumentaron levemente las temperaturas mínimas a $7,5^{\circ} \mathrm{C}$. con ausencia de heladas. La incidencia de Botrytis $\mathrm{cn}$ hojas aumenta un 13\% con respecto al mes de agosto, alcanzando el $49 \%$ de los árboles (Figura 1). En frutos la incidencia se duplica llegando al $30 \%$ de los árboles muestreados.

Por último en octubre las condiciones ambientales se mantienen similares a las del mes anterior, pero con un alza en las temperaturas máximas medias Ilegando a los $23^{\circ} \mathrm{C}$ y una leve diminución en la humedad relativa. La incidencia de Botrytis en árboles alcanzal el 69\%. la mayor del período cstudiado (Figura 1), esto probablemente se explica por las reinfecciones ocurridas, sumándose a las favorables condiciones ambientales para el desarrollo del hongo analizadas anteriormente. En frutos la incidencia disminuye a un $24 \%$ lo que es difícil de explicar, pues coincide con su clapa de mayor susceptibilidad al ataque del patógeno. Probablemente se deba al inicio de las labores de cosecha, en que se colectan los frutos más maduros, o quizás a la mayor ventilación dentro de los árboles debido al inicio del periodo de renoración del follaje. Esto concuerda con Thomas \& Marois (1986). quicnes señalan que no se desarrolla micelio externo en las bayas de vid expuestas al vicnto. Morales (1992), recomienda la ventilación en parrones de vides por medio de deshojes, para disminuir las micosis, produciendo un rápido desecamiento de las superficies de las plantas.

En las semillas de los fritos colectados en el mes de octubre, no se desarrolló Botrytis, por lo que podría pensarse que el hongo no permanece latente o no invade las scmillas.

En las flores colcctadas en febrero ni en flores tardías, creció Botrytis y tampoco se desarrolló a partir de restos florales presentes en frutos pequeños durante el mes de abril, por lo que también podría descartarse como fuente de inóculo para los frutos. Esto probablemente se deba a las condiciones ambientales desfavorables al desarrollo de Botrytis, características de la zona de Quillota durante encro y febrero, donde la humedad relativa es alta pero las iemperaturas mínimas son demasiado altas como 

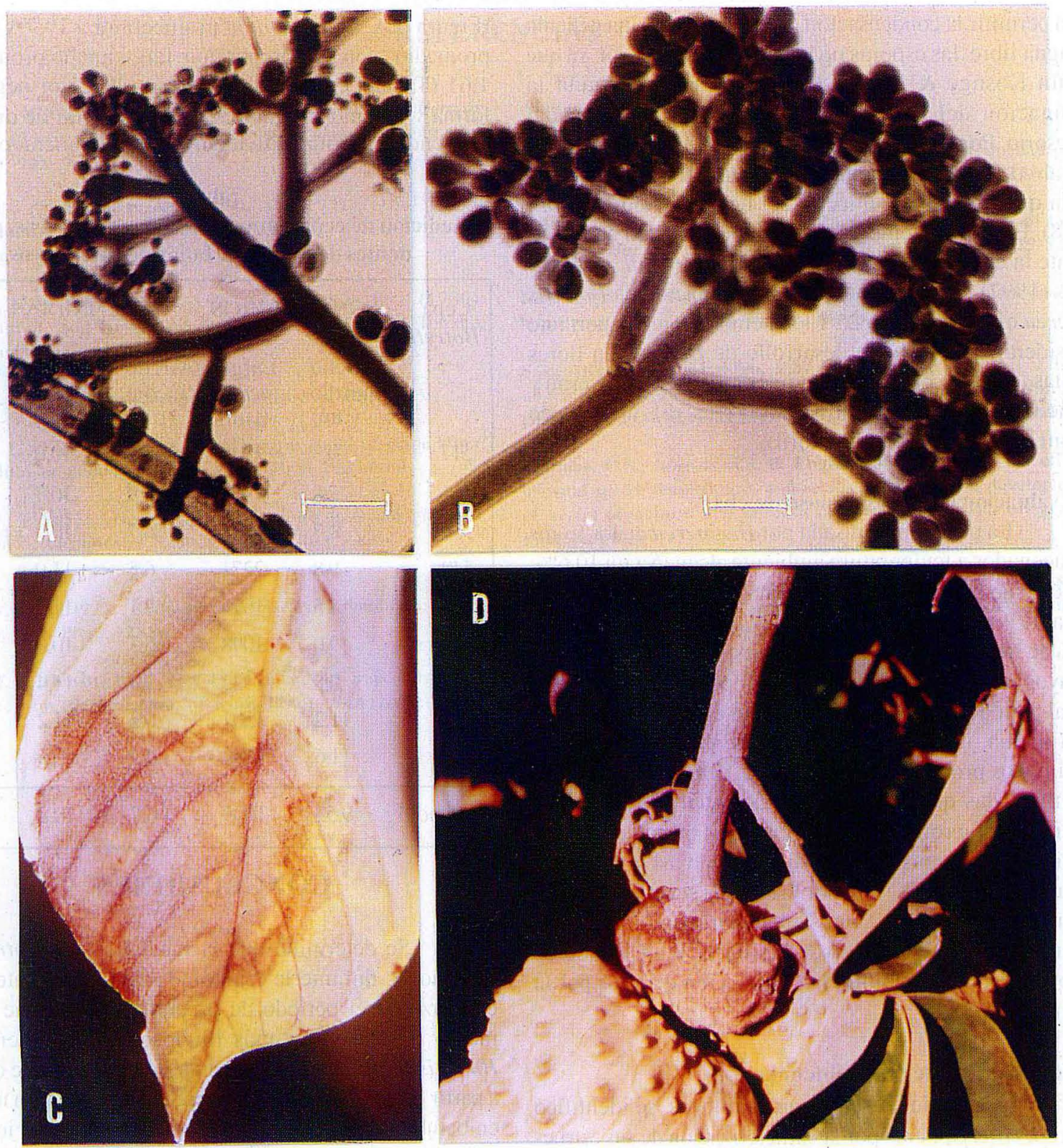

Figura. 2. Botrytis cinerea: a) Conidióforo y jóvenes vesículas iniciando la formación de conidios. b) Conidióforos y conidios maduros. c) Hoja con signos de pudrición gris en el árbol. d) Fruto con pudrición en el árbol. Barra $=20 u m$. 
para permitir la condensación. En ausencia de una pelieula de agua libre. las esporas no germinan ni penetran. ya que según Kosuge \& Hewitl (196t). el agua estimula la clongación del tubo germinativo y la formación del apresorio. Por otro lado las temperaturas máximas estur icron alrededor de $28^{\circ} \mathrm{C}$. mus cerca de los $36^{\circ} \mathrm{C}$. temperatura en la que no crece el micelio del hongo (Thomas et al.. 1988). Estas altas temperaturas lambién afectan negativamente la esporulación. pues Sosa-Alvares et al. (1995)y Ellis (1997). cncontraron que la esporulación de Botrytis cinerea cs muy pobre a $25^{\circ \prime} \mathrm{C}$. sin cmbargo. es importante considerar que no hubo desarrollo de Botrytis en flores tardías, es decir. cuando las temperaturas disminuycron y aumentó la humedad relativa (abril y maỵo). ni tampoco colonizando restos florales (abril).

\section{b) Evaluación de frutos en pośteosechal.}

La pudrición causada por B. cinceréa en los frutos almacenados a temperaltura ambiente alcaur ó a un 10.6\%. sicndo bajo las condiciones de almacenaje dadas. más importante la pudrición causiada por Rhizopus que alcan\%ó al $53 \%$ de los frutos. De acuerdo a resultados obtenidos en ensayos previos. ésla no es clevadia. posiblemente debido al periodo de secjuia de los últimos cualtro años. Durante la secquia. la incidencia de $B$. cinerea en el huerto de chirimoyos probablemente fue bajal. debido a que las hojas infectadas que caen al suelo y guardan el micelio invernante. fueron menores en cantidad gue en un año de precipitaciones normales. disminuyendo asi el inóculo ( Braun \& Sutton. 1987). y por lo tanto la severidad de csta enfermedad en postcosecha. Sin embargo. se debe considerar que igualmente el nivel de infección alcanzado en frutos en csla clapa. limila la cxportación de ćstos por la via marítima.

\section{c) Identificación de ceparas obtenidas.}

La especie presente en chirimoros. se identificó como 13. cincerea Pers. por la caracterización de sus estructuras reproductivas desde el punto de vista macro y microscópico. Las colonias de B. cineréa cultivadas en medio APDA. presentaron un micelio blancuecino quic luego se tornaba parclo - grisácco. Sc observó al microscopio óptico que el micelio posec septos y liene crecimiento ramilicado. Los conidióforos de las 9 cepas analizadias producto de los muestreos fucron medidos al microscopio óptico encontrándose un promedio de $1.6(1) 5 \mu m$ de largo y $9.52 \mu \mathrm{m}$ de ancho. Las medidas promedio de cada cepa se encuentran en la Tabla I. Los conidióforos se presentan en forma derecha o flexuosa. lisos. de color calé. ramificados dico o tricotomicamente. con ramas generalmente restringidas a las regiones apicales. que en sus extremos tienden a dilatarse formando una ampulla conidiógena pílida (Figuras $2 \mathrm{a}$ y $2 \mathrm{~b}$ ). Conidios lisos. calfé pálidos. oboroides
(Figura 2b). con un hilum protuberante. 10.7 $\times 6.7 \mu \mathrm{m}$ promedio . con una proporción largo/ancho promedio de 1.61 (Tabla 1). Los esclerocios son de color negro y de formal y tamaño irregulares. de 1 a 5 mm de diámetro. creciendo en forma individual o en conglomerados

Tabla 1

Medición de cepas obtenidas en los muestreos, procedentes de tejidos de hojas de chirimoyos.

\begin{tabular}{|c|c|c|c|c|c|}
\hline \multirow{2}{*}{$\begin{array}{l}\text { Cepa } \\
\text { Botrytis }\end{array}$} & \multicolumn{2}{|c|}{ CONIIIOEFOOROS } & \multicolumn{3}{|c|}{ CONIIIAS } \\
\hline & $\begin{array}{r}\text { Ancho } \\
\text { medio } \\
(\text { fum })\end{array}$ & $\begin{array}{r}\text { Largo } \\
\text { Imedio } \\
(\text { (1III) }\end{array}$ & $\begin{array}{r}\text { Anclio } \\
\text { medio } \\
\text { (jum) }\end{array}$ & $\begin{array}{r}\text { Largo } \\
\text { medio } \\
\text { (flun) }\end{array}$ & Rayón \\
\hline 517 & 9.3 & $1.3+1)$ & 6.7 & 10.0 & 1.5() \\
\hline 521 & 7.8 & 1974 & 6.9 & 10.7 & 1.57 \\
\hline 539 & 8.7 & 1878 & 6.7 & 10.9 & 1.63 \\
\hline 54() & 9.8 & 2271 & 6.5 & 11.0 & 1.71 \\
\hline $5+1$ & 8.5 & 1675 & 7.1 & 11.5 & 1.63 \\
\hline 542 & $9.1)$ & 1098 & 6.3 & $10 .(1)$ & 1.59 \\
\hline 54.3 & 9.8 & 1522 & 7.4 & 11.4 & 1.54 \\
\hline 54 & 11.2 & 15() 4 & 6.2 & 11.2 & 1.81 \\
\hline $5+6$ & 11.6 & 1180 & 6.2 & 9.6 & 1.55 \\
\hline Promedio & 9.52 & $16(x) 5$ & 6.7 & 10.7 & 1.61 \\
\hline
\end{tabular}

\section{CONCLUSIONES}

Se determinó que la incidencia de Botrytis en chirimoyos durante la temporada de crecimiento 1997. connenzó en el período de precipilaciones y fue en aumento en los meses más lluviosos. La incidencia de Botrytis en árboles. medida en tejido de hojas. se obserró a partir del mes de mayo y aumentó hasta lograr un máximo en octubre. Un mes mas tarde que en hojas. en junio. se presentó en frutos y la máxima incidencia se observó en el mes de septiembre. Los órganos del chirimovo afectados por Botrytis son họjas y frutos. No se detectó la presencia de Botrytis en flores en ninguno de los muestreos. Tampoco se detectó presencia de Borrytis en el interior de semillas sembradas en APDA. La incidencia de Botrytis durante la postcosecha alcallizó sólo al $10.6 \%$ de los frutos y de los ) aislimmicntos obtenidos de hojas a lo largo de la temporada se determinó sólo la presencia de B. cineréa.

\section{AGRADECIMIIENTOS}

Las autoras agradecen al Dr E.Piontelli por la colaboración prestada en la identificación de ecpas de $B$. cincrea. Al mismo tiempo. a Gladys Andrade y Guillermo Durán por su asistoncia técnicia. 


\section{REFERENCIAS}

Braun, P.(;. \& Sutton, J.('. (1987). Inuculum sources of Botrytis cinerea in truit rot of strawherries in () nario. Canadian Joumal of Plant Patholog! $9: 1-5$

Cortesi, P.; Kerbetto, F. \& Vercesi, A (199)). Biologia ed epidemiologia di Botrytis cinerea Pers. su vite. Inlormatore Fitopitologico $6:+5-48$

Domsch, K.H. (iams, W. \& Anderson,T-lI. (1980). Compendium of Soil lungi. Academic l'ress. I.ondon .N.Y.Toronto, Sydney. San linancisco.

Ellis, M.13. (1971). Dematiacious Hyphomycess. CMII, Kew:

Ellis,M.A. (1997). Botrytis bunch rot or gray mold of grape http:/ www:ag.ohio-state.eduohioline hyg-fact/30()0/3025.html

Itec-Chile. (1985). Investigación y desarollo de kconologias para exportacion de chirimoya. Santiago. Intec-C'hile. Sp.

Jarvis, W.R. (1992). Managing discases in greenhouse crops. Minnesotil. APS Press. $28 \times p$

Kosuge, T. \& Ilewitt, W.13. (1964). Exudiates of grape berries and their ellect on gemination of conidia of Borrytis cinerea.
Phỵtopathology 54:167-172

Morales, A. (1992). Borryis cinerea Pers.: una enlemedad para prevenir. Prevencion versus control curativo. Aconex 35:21-25

Nelson, K.E. (1951). Factors influencing the infection of table grapes by Botrytis cincea (Pers). Phytopathology 41:319-326

Ries, S. (1995). Gray mold of strawberry. Report on plant disease. http://www.aces.uius.edu/-ipm/fruits/rpds/704/704.html

Sosa-Alvarez, M.; Madden, L.V. \& Ellis, M.A. (1995). Eflects of temperature and wetness duration on sporulation of Botrytis cinerea on strawberry leaf residues. Plant Disease 79:609-615

Thomas, C.S.; Marois, J.J. \& English, J.T. (1988). The effects of wind speed, temperature, and relative humidity on development of acrial myeclium and conidia of Borrytis cinerea on grape. Phytopathology 78:260-265

Thomas, C..S. \& Marois, J.J. (1986). Effect of wind and relative humidity on sporulation and external mycelium formation of Botrytis on grape. Phytopathology 76:1114 